TRANSACTIONS OF THE

AMERICAN MATHEMATICAL SOCIETY

Volume 358, Number 9, September 2006, Pages 4119-4134

S 0002-9947(06)03842-6

Article electronically published on February 20, 2006

\title{
THE MONOMIAL IDEAL OF A FINITE MEET-SEMILATTICE
}

\author{
JÜRGEN HERZOG, TAKAYUKI HIBI, AND XINXIAN ZHENG
}

\begin{abstract}
Squarefree monomial ideals arising from finite meet-semilattices and their free resolutions are studied. For the squarefree monomial ideals corresponding to poset ideals in a distributive lattice, the Alexander dual is computed.
\end{abstract}

\section{INTRODUCTION}

One of the most influential results in classical lattice theory is Birkhoff's fundamental structure theorem for finite distributive lattices 10, Theorem 3.4.1], which guarantees that, given a finite distributive lattice $\mathcal{L}$, there is a unique poset (partially ordered set) $P$ such that $\mathcal{L}$ is isomorphic to the poset $\mathcal{J}(P)$ consisting of all poset ideals (including the empty set) of $P$, ordered by inclusion. (A poset ideal of $P$ is a subset $I \subset P$ with the property that if $p \in I$ and $q \in P$ with $q \leq p$, then $q \in I$.) In fact, if $P$ is the subposet of $\mathcal{L}$ consisting of all join-irreducible elements of $\mathcal{L}$, then $\mathcal{L}=\mathcal{J}(P)$. (An element $p \in \mathcal{L}$ with $p \neq \hat{0}$ is called join-irreducible if there is no $q, r \in \mathcal{L}$ with $q<p$ and $r<p$ such that $p=q \vee r$.) In other words, by identifying $\mathcal{L}$ with $\mathcal{J}(P)$, if $p \in \mathcal{L}$ and $I=\{q \in P: q \leq p\} \in \mathcal{J}(P)$, then $p=I$.

Fix a finite distributive lattice $\mathcal{L}=\mathcal{J}(P)$. Let $K$ be a field and let $S=$ $K\left[\left\{x_{p}, y_{p}\right\}_{p \in P}\right]$ be the polynomial ring in $2|P|$ variables over $K$ with $\operatorname{deg} x_{p}=1$ and $\operatorname{deg} y_{p}=1$ for all $p \in P$. We associate each element $I \subset \mathcal{J}(P)=\mathcal{L}$ with the squarefree monomial $u_{I}=\left(\prod_{p \in I} x_{p}\right)\left(\prod_{p \in P \backslash I} y_{p}\right) \in S$. In the previous paper 6 ] the monomial ideal $H_{\mathcal{L}}=\left(u_{I}\right)_{I \in \mathcal{L}}$ is discussed from viewpoints of both combinatorics and commutative algebra. The purpose of the present paper is to introduce the squarefree monomial ideal $H_{\mathcal{L}}$ for an arbitrary finite meet-semilattice $\mathcal{L}$ and to generalize some of the results obtained in [6].

Now, let $\mathcal{L}$ be an arbitrary finite meet-semilattice [10, p. 103] and let $P \subset \mathcal{L}$ be the set of join-irreducible elements of $\mathcal{L}$. For each element $q \in \mathcal{L}$ we write $\ell(q)=\{p \in P: p \leq q\} \subset P$. In particular $\ell(\hat{0})=\emptyset$. Note that $\ell(q)$ is a poset ideal of $P$, and that $q \in \ell(q)$ if and only if $q$ is join-irreducible. We thus obtain the map $\ell: \mathcal{L} \rightarrow \mathcal{B}_{P}$, which we call the canonical embedding of $\mathcal{L}$ into the Boolean lattice $\mathcal{B}_{P}$ consisting of all subsets of $P$ ordered by inclusion. As in the case of finite distributive lattices explained in the previous paragraph, let $K$ be a field and $S=K\left[\left\{x_{p}, y_{p}\right\}_{p \in P}\right]$ the polynomial ring in $2|P|$ variables over $K$ with $\operatorname{deg} x_{p}=1$ and $\operatorname{deg} y_{p}=1$ for all $p \in P$. We associate each element $q \in \mathcal{L}$ with the squarefree monomial $u_{q}=\left(\prod_{p \in \ell(q)} x_{p}\right)\left(\prod_{p \in P \backslash \ell(q)} y_{p}\right) \in S$ and set $H_{\mathcal{L}}=\left(u_{q}\right)_{q \in P} \subset S$.

Received by the editors November 6, 2003 and, in revised form, September 2, 2004 and September 9, 2004.

2000 Mathematics Subject Classification. Primary 13D02, 13H10, 06A12, 06 D99. 
In the present paper the following topics on squarefree monomial ideals $H_{\mathcal{L}}$ arising from finite meet-semilattices $\mathcal{L}$ will be studied:

- When has the squarefree monomial ideal $H_{\mathcal{L}}$ a linear resolution? Theorem 1.3 guarantees that $H_{\mathcal{L}}$ has a linear resolution if and only if $\mathcal{L}$ is meetdistributive. (A finite meet-semilattice $\mathcal{L}$ is called meet-distributive if each interval $[x, y]=\{p \in \mathcal{L}: x \leq p \leq y\}$ of $\mathcal{L}$ such that $x$ is the meet of the lower neighbors of $y$ in this interval is Boolean. Here we call $z$ a lower neighbor of $y$ if $y$ covers $z$.)

- How can we construct a finite multigraded free $S$-resolution $\mathbb{F}$ of $H_{\mathcal{L}}$ ? A construction of such a finite free resolution is given in Theorem [2.1)(a). Moreover, we will characterize when our resolution is minimal. In fact, it will be proved in Theorem 2.1(b) that our resolution is minimal if and only if, for any $p \in \mathcal{L}$ and for any proper subset $S \subset N(p)$, the meet $\bigwedge\{q: q \in S\}$ is strictly greater than the meet $\bigwedge\{q: q \in N(p)\}$, where $N(p)$ is the set of lower neighbors of $p$ in $\mathcal{L}$. In particular, if $\mathcal{L}$ is a meet-distributive meetsemilattice, then our finite free resolution is minimal (Corollary 2.2). On the other hand, when $\mathcal{L}$ is a meet-distributive meet-semilattice, the differential $\partial$ in the finite multigraded free $S$-resolution $\mathbb{F}$ of $H_{\mathcal{L}}$ obtained in Theorem 2.1(a) will be described (Theorem 3.1).

- Since $H_{\mathcal{L}}$ is a squarefree monomial ideal, there is a simplicial complex $\Delta$ whose Stanley-Reisner ideal $I_{\Delta}$ coincides with $H_{\mathcal{L}}$. We are interested in the Alexander dual $\Delta^{\vee}$ of $\Delta$. In the case that $\mathcal{L}$ is a finite distributive lattice, a nice description of $\Delta^{\vee}$ can be obtained ([6, Lemma 3.1]). It seems, however, rather difficult for an arbitrary finite meet-semilattice to obtain an explicit description of the Alexander dual of $H_{\mathcal{L}}$. We will consider a special meet-distributive meet-semilattice, namely, a poset ideal $\mathcal{I}$ of a finite distributive lattice. In this case the Alexander dual of $H_{\mathcal{I}}$ will be studied in Theorem 4.2. Moreover, since $H_{\mathcal{I}}$ has a linear resolution, it follows that the Alexander dual of $H_{\mathcal{I}}$ is Cohen-Macaulay. Such CohenMacaulay complexes will be discussed in Theorem 4.3 .

\section{Algebraic characterizations OF MEET-DISTRIBUTIVE MEET-SEMILATTICES}

Let $\mathcal{L}$ be an arbitrary finite meet-semilattice (cf. 10, p. 103]), and let $P \subset \mathcal{L}$ be the set of join-irreducible elements of $\mathcal{L}$. We denote by $\hat{0}$ and $\hat{1}$ the minimal and maximal element of $\mathcal{L}$. (Since $\mathcal{L}$ is a finite meet-semilattice, it follows 10 , Proposition 3.3.1] that $\mathcal{L}$ possesses $\hat{1}$ if and only if $\mathcal{L}$ is a lattice.) Recall that $p \in \mathcal{L}$ is join-irreducible if $p \neq \hat{0}$ and $p$ is not a join of elements strictly less than $p$.

To each element $p \in \mathcal{L}$ we associate the subset $\ell(p)=\{q \in P: q \leq p\}$ of $P$. Note that $p \in \ell(p)$ if and only if $p$ is join irreducible. In any case, $\ell(p)$ is a poset ideal of $P$. Recall that a poset ideal of $P$ is a subset $I \subset P$ such that if $r \in I$ and $t \leq r$, then $t \in I$. The set of generators of $I$ is the set of maximal elements in $I$, denoted by $G(I)$.

We obtain a map

$$
\ell: \mathcal{L} \longrightarrow \mathcal{B}_{P}
$$

which we call the canonical embedding into the Boolean lattice $\mathcal{B}_{P}$ consisting of all subsets of $P$ ordered by inclusion. 
We call the cardinality of $\ell(p)$ the degree of $p$, and denote it by $\operatorname{deg} p$. One always has the inequality $\operatorname{rank} p \leq \operatorname{deg} p$. Recall that the rank of $p$ is the maximal length of chains descending from $p$.

Lemma 1.1. Let $\mathcal{L}$ be a finite meet-semilattice, $\ell$ the canonical embedding and $s, t \in \mathcal{L}$ any two elements. We have

(i) $s=t$ if and only if $\ell(s)=\ell(t)$;

(ii) $s \leq t$ if and only if $\ell(s) \subseteq \ell(t)$;

(iii) $\ell(s) \cap \ell(t)=\ell(s \wedge t)$.

Proof. Note that each element of $\mathcal{L}$ is the join of elements in $P$. From this observation all assertions follow.

The lemma implies that $\ell$ is an injective order preserving map. In general however, $\ell$ is not an embedding of lattices. It is not difficult to see that $\ell$ is an embedding of meet-semilattices if and only if $\mathcal{L}$ is meet-distributive.

We now introduce the definition of meet-distributive meet-semilattices. A finite meet-semilattice $\mathcal{L}$ is called meet-distributive if each interval $[x, y]=\{p \in \mathcal{L}: x \leq$ $p \leq y\}$ of $\mathcal{L}$ such that $x$ is the meet of the lower neighbors of $y$ in this interval is Boolean. Here we call $z$ a lower neighbor of $y$ if $y$ covers $z$.

The following combinatorial characterization of meet-distributive lattices are discussed in the survey article [4. A finite meet-semilattice is called graded if for each element all of its maximal chains have the same length.

Lemma 1.2. For a finite lattice $\mathcal{L}$ the following conditions are equivalent:

(i) $\mathcal{L}$ is meet-distributive;

(ii) $\mathcal{L}$ is graded and $\operatorname{deg} \hat{1}=\operatorname{rank} \hat{1}$;

(iii) $\mathcal{L}$ is graded and $\operatorname{deg} \hat{p}=\operatorname{rank} \hat{p}$ for all $p \in \mathcal{L}$;

(iv) each element in $\mathcal{L}$ is a unique minimal join of join-irreducible elements;

(v) $\mathcal{L}$ is lower semimodular, and any upper semimodular sublattice is distributive.

We now introduce the squarefree monomial ideal $H_{\mathcal{L}}$ associated with a finite meet-semilattice $\mathcal{L}$. Let $P$ be the set of join irreducible elements of $\mathcal{L}$. Let $K$ be a field and $S=K\left[\left\{x_{p}, y_{p}\right\}_{p \in P}\right]$ the polynomial ring in $2|P|$ variables over $K$. For each element $q \in \mathcal{L}$ write

$$
u_{q}=\prod_{p \in \ell(q)} x_{p} \prod_{p \in P \backslash \ell(q)} y_{p}
$$

and set $H_{\mathcal{L}}=\left(u_{q}\right)_{q \in \mathcal{L}}$.

Note that height $\left(H_{\mathcal{L}}\right)=2$ if $\mathcal{L}$ is a lattice. In fact, $H_{\mathcal{L}} \subset\left(x_{p}, y_{p}\right)$ for any $p \in P$, while on the other hand $u_{\hat{0}}=\prod_{p \in P} y_{p}$ and $u_{\hat{1}}=\prod_{p \in P} x_{p}$ both belong to $H_{\mathcal{L}}$ and have no common factor.

Let $I$ be a monomial ideal with the (unique) minimal set $G(I)$ of monomial generators. The ideal $I$ is said to have linear quotients if the elements of $G(I)$ can be ordered $u_{1}, \ldots, u_{m}$ such that the colon ideals $\left(u_{1}, \ldots, u_{i-1}\right): u_{i}$ are generated by variables. If $I$ is squarefree, then $I$ has linear quotients if and only if for each $i$ and each $j<i$ there exists $k<i$ such that $u_{k} /\left[u_{k}, u_{i}\right]$ is a variable and divides $u_{j}$. Here $[u, v]$ denotes the greatest common divisor of $u$ and $v$.

It is easy to see that if all generators of $I$ have the same degree, and $I$ has linear quotients, then $I$ has a linear resolution. 
We now come to our algebraic characterization of meet-distributive meet-semilattices.

Theorem 1.3. Let $\mathcal{L}$ be an arbitrary finite meet-semilattice. The following conditions are equivalent:

(i) $\mathcal{L}$ is meet-distributive;

(ii) $H_{\mathcal{L}}$ has linear quotients;

(iii) $H_{\mathcal{L}}$ has a linear resolution;

(iv) $H_{\mathcal{L}}$ has linear relations.

Proof. (i) $\Rightarrow$ (ii): We fix a linear order $\prec$ on $\mathcal{L}$ which extends the partial order given by the degree. We put $u_{r}<u_{q}$ if $r \prec q$. For any $u_{q} \in H_{\mathcal{L}}$ and any $u_{r}<u_{q}$, let $t$ be a lower neighbor of $q$ in the interval $[r \wedge q, q]$. Then $u_{t} /\left[u_{t}, u_{q}\right]=y_{p}$, where $\{p\}=\ell(q) \backslash \ell(t)$. We claim that $y_{p}$ divides $u_{r}$. If not, then $x_{p}$ divides $u_{r}$ and so $p \in \ell(r) \cap \ell(q)=\ell(r \wedge q)$. Thus $p \in \ell(t)$, since $r \wedge q \leq t$.

(ii) $\Rightarrow$ (iii) and (iii) $\Rightarrow$ (iv) are trivial.

(iv) $\Rightarrow$ (i): Suppose $\mathcal{L}$ is not meet-distributive. Then by Lemma 1.2(iii) (which is also valid if $\mathcal{L}$ is a meet-distributive meet-semilattice) there exist $p, q \in \mathcal{L}$ such that $q$ is the lower neighbor of $p$ and $\operatorname{deg} p-\operatorname{deg} q>1$. The ideal $\left(u_{p}, u_{q}\right)$ is generated by precisely those monomials in $G\left(H_{\mathcal{L}}\right)$ which are not divided by $x_{r}$ for all $r \in P \backslash \ell(p)$, and are not divided by all $y_{s}$ for all $s \in \ell(q)$. Since we assume that $H_{\mathcal{L}}$ has linear relations, the restriction lemma in [7, Lemmma 4.4] implies that $\left(u_{p}, u_{q}\right)$ has linear relations, contradicting the fact that $\operatorname{deg} p-\operatorname{deg} q>1$.

Corollary 1.4. Let $\mathcal{L}$ be a finite upper semimodular lattice. Then the following conditions are equivalent:

(i) $H_{\mathcal{L}}$ has a linear resolution;

(ii) $\mathcal{L}$ is distributive.

Proof. The assertion follows from Lemma 1.2(v) and Theorem 1.3 .

Let $\Delta$ be a simplicial complex on the vertex set $[n]=\{1, \ldots, n\}$. The simplicial complex

$$
\Delta^{\vee}=\{[n] \backslash F: F \notin \Delta\}
$$

is called the Alexander dual of $\Delta$. It is easy to see that $\left(\Delta^{\vee}\right)^{\vee}=\Delta$.

A vertex cover of $\Delta$ is a set $G \subset[n]$ such that $G \cap F \neq \emptyset$ for all $F \in \mathcal{F}(\Delta)$, where $\mathcal{F}(\Delta)$ denotes the set of facets (maximal faces) of $\Delta$. A vertex cover is called minimal if it is minimal with respect to inclusion. We also denote by $\mathcal{C}(\Delta)$ the set of minimal vertex covers of $\Delta$.

As usual we denote by $I_{\Delta}$ the Stanley-Reisner ideal of $\Delta$. The facet ideal is defined to be

$$
I(\Delta)=\left(x_{F}: F \in \mathcal{F}(\Delta)\right)
$$

where $x_{F}=\prod_{i \in F} x_{i}$.

For $F=\left\{i_{1}, \ldots, i_{k}\right\} \subset[n]$ set $P_{F}=\left(x_{i_{1}}, \ldots, x_{i_{k}}\right)$, and let $\Gamma$ be the unique simplicial complex such that $I_{\Delta}=I(\Gamma)$. Then

$$
I_{\Delta}=\bigcap_{F \in \mathcal{C}(\Gamma)} P_{F} \quad \text { and } \quad I_{\Delta \vee}=\left(x_{F}: F \in \mathcal{C}(\Gamma)\right) .
$$


Set $F^{c}=[n] \backslash F$ and

$$
\Delta^{c}=\left\langle F^{c}: F \in \mathcal{F}(\Delta)\right\rangle
$$

Then

$$
I_{\Delta} \vee=I\left(\Delta^{c}\right) .
$$

The following lemma gives important algebraic properties of Alexander duality.

Lemma 1.5. Let $K$ be a field, $\Delta$ a simplicial complex, $I_{\Delta}$ the Stanley-Reisner ideal and $K[\Delta]$ the Stanley-Reisner ring of $\Delta$. Then:

(i) (Eagon-Reiner [3]) $K[\Delta]$ is Cohen-Macaulay $\Longleftrightarrow I_{\Delta} \vee$ has a linear resolution.

(ii) ([7]) $\Delta$ is shellable $\Longleftrightarrow I_{\Delta \vee}$ has linear quotients.

Theorem 1.3 together with Lemma 1.5 yields

Corollary 1.6. Let $\mathcal{L}$ be an arbitrary finite meet-semilattice, and let $\Delta_{\mathcal{L}}$ be the simplicial complex whose Stanley-Reisner ideal is $H_{\mathcal{L}}$. The following conditions are equivalent:

(i) $\left(\Delta_{\mathcal{L}}\right)^{\vee}$ is shellable;

(ii) $\left(\Delta_{\mathcal{L}}\right)^{\vee}$ is Cohen-Macaulay;

(iii) $\mathcal{L}$ is meet-distributive.

Proposition 1.7. Let $\mathcal{L}$ be a finite lattice and $P$ its poset of join irreducible elements. Then

(i) the minimal prime ideals of height 2 of $H_{\mathcal{L}}$ are $\left(x_{p}, y_{q}\right)$, where $p, q \in P$ and $p \leq q$

(ii) $H_{\mathcal{L}}$ has only height 2 minimal prime ideals if and only if $\mathcal{L}$ is distributive.

Proof. Let $\hat{\mathcal{L}}$ be the distributive lattice consisting of all poset ideals of $P$. Then $\ell$ induces an injective order preserving map $\ell: \mathcal{L} \rightarrow \hat{\mathcal{L}}$. Thus $H_{\mathcal{L}} \subset H_{\hat{\mathcal{L}}}$, and equality holds if and only if $\mathcal{L}$ is distributive. This follows from Birkhoff's fundamental structure theorem 10 .

(i) The minimal prime ideals of $H_{\hat{\mathcal{L}}}$ are precisely the ideals $\left(x_{p}, y_{q}\right)$, where $p, q \in$ $P$ and $p \leq q$; see [6]. Of course these are also minimal prime ideals of $H_{\mathcal{L}}$. We claim that there are no other minimal prime ideals of height 2 of $H_{\mathcal{L}}$. Indeed, any such ideal must contain some $x_{p}$ and some $y_{q}$, since $\prod_{p \in P} x_{p}$ and $\prod_{p \in P} y_{p}$ belong to $H_{\mathcal{L}}$. Suppose $p \not \leq q$; then $u_{q}$ is not contained in $\left(x_{p}, y_{q}\right)$.

(ii) It remains to show that if $\mathcal{L}$ is not distributive, then there exists a minimal prime ideal of $H_{\mathcal{L}}$ of height $>2$. In fact, the proof of (i) shows that if such a minimal prime ideal does not exist, then $H_{\mathcal{L}}=H_{\hat{\mathcal{L}}}$. Therefore $\mathcal{L}=\hat{\mathcal{L}}$, and hence $\mathcal{L}$ is distributive.

Recall that a simplicial complex is called flag if each minimal nonface consists of two elements. Proposition 1.7 together with (11) implies

Corollary 1.8. A finite lattice $\mathcal{L}$ is distributive if and only if $\left(\Delta_{\mathcal{L}}\right)^{\vee}$ is flag. 


\section{A free Resolution of $H_{\mathcal{L}}$}

The main theorem of the present section is the following.

Theorem 2.1. Let $\mathcal{L}$ be a finite meet-semilattice.

(a) There exists a finite multigraded free $S$-resolution $\mathbb{F}$ of $H_{\mathcal{L}}$ such that for each $i \geq 0$, the free module $F_{i}$ has a basis with basis elements

$$
b(p ; S),
$$

where $p \in \mathcal{L}$ and $S$ is a subset of the set of lower neighbors $N(p)$ of $p$ with $|S|=i$. The multidegree of $b(p ; S)$ is the least common multiple of $u_{p}$ and all monomials $u_{q}$ with $q \in S$.

(b) The following conditions are equivalent:

(i) the resolution constructed in (a) is minimal;

(ii) for any $p \in \mathcal{L}$ and any proper subset $S \subset N(p)$ the meet $\bigwedge\{q: q \in S\}$ is strictly greater than the meet $\bigwedge\{q: q \in N(p)\}$.

We call a finite meet-semilattice satisfying condition (b)(ii) meet-irredundant.

Proof of Theorem 2.1. (a) The resolution will be built by an iterated mapping cone construction. As in the proof of Theorem 1.3 we fix a linear order $\prec$ on $\mathcal{L}$ which extends the partial order given by the degree. For any $p$ in $\mathcal{L}$ we inductively construct a complex $\mathbb{F}(p)$ which is a multigraded free $S$-resolution of the ideal $H_{\mathcal{L}}(p)$ generated by all $u_{q} \in H_{\mathcal{L}}$ with $q \preceq p$. Then $\mathbb{F}(q)$ is the desired resolution, where $q \in \mathcal{L}$ is the maximal element with respect to $\prec$.

The complex $\mathbb{F}(\hat{0})$ is defined as $F_{i}(\hat{0})=0$ for $i>0$, and $F_{0}(\hat{0})=S$. This complex, together with the augmentation map $\varepsilon: S \rightarrow H_{\mathcal{L}}(\hat{0}), 1 \mapsto u_{\hat{0}}$, is a free resolution of $H_{\mathcal{L}}(\hat{0})$.

Now let $p \in \mathcal{L}, p \neq \hat{0}$, and let $q \in \mathcal{L}, q \prec p$ be the element preceding $p$. Then $H_{\mathcal{L}}(p)=\left(H_{\mathcal{L}}(q), u_{p}\right)$, and hence we get an exact sequence of multigraded $S$-modules

$$
0 \longrightarrow(S / L)\left(- \text { multideg } u_{p}\right) \longrightarrow S / H_{\mathcal{L}}(q) \longrightarrow S / H_{\mathcal{L}}(p) \longrightarrow 0,
$$

where $L$ is the colon ideal $H_{\mathcal{L}}(q): u_{p}$. As in the proof of Theorem 1.3 one shows that

$$
L=\left(\left\{u_{t} /\left[u_{t}, u_{p}\right]\right\}_{t \in N(p)}\right) .
$$

Let $\mathbb{T}$ be the Taylor complex associated with the monomials $u_{t} /\left[u_{t}, u_{p}\right], t \in N(p)$; see [5]. Then $\mathbb{T}$ is a multigraded free resolution of $S / L$ with $T_{0}=S, T_{1}=$ $\bigoplus_{t \in N(p)} S e_{t}$ and $T_{i}=\bigwedge^{i} T_{0}$ for $i \geq 1$. Thus $T_{i}$ has a basis whose elements are $e_{t_{1}} \wedge e_{t_{2}} \wedge \cdots \wedge e_{t_{i}}$ with $t_{1}<t_{2}<\cdots<t_{i}$. The multidegree of $e_{t_{1}} \wedge e_{t_{2}} \wedge \cdots \wedge e_{t_{i}}$ is the least common multiple of the elements $u_{t_{j}} /\left[u_{t_{j}}, u_{p}\right], j=1, \ldots, i$.

The shifted complex

$$
\mathbb{T}\left(- \text { multideg } u_{p}\right)
$$

is a multigraded free resolution of $(S / L)\left(-\right.$ multideg $\left.u_{p}\right)$. We denote the basis element of $T_{i}\left(-\right.$ multideg $\left.u_{p}\right)$ which corresponds to $e_{t_{1}} \wedge e_{t_{2}} \wedge \cdots \wedge e_{t_{i}}$ by $b\left(p ;\left\{t_{1}, \ldots, t_{i}\right\}\right)$. Then multideg $b\left(p ; t_{1}, \ldots, t_{i}\right)=$ multideg $u_{p}+$ multideg $e_{t_{1}} \wedge e_{t_{2}} \wedge \cdots \wedge e_{t_{i}}$, and hence it is the least common multiple of $u_{p}, u_{t_{1}}, \ldots, u_{t_{i}}$.

The monomorphism $(S / L)\left(-\right.$ multideg $\left.u_{p}\right) \rightarrow S / H_{\mathcal{L}}(q)$ induces a comparison map

$$
\alpha: \mathbb{T}\left(- \text { multideg } u_{p}\right) \longrightarrow \mathbb{F}(q)
$$


of multigraded complexes. We let $\mathbb{F}(p)$ be the mapping cone of $\alpha$. Then $\mathbb{F}(p)$ is a multigraded free $S$-resolution of $H_{\mathcal{L}}(p)$, and has the desired multigraded basis.

(b)(i) $\Rightarrow$ (ii): Let $p \in \mathcal{L}$ with $|N(p)|>1$, and let $S \subset N(p)$ be a subset. By the definition of the differential $\partial$ of $\mathbb{F}$ we have

$$
\partial b(p ; S)=\sum_{q \in S} \pm v_{q} b(p ; S \backslash\{q\})+\cdots,
$$

where $v_{q}=\operatorname{multideg} b(p ; S) /$ multideg $b(p ; S \backslash\{q\})$. Therefore the resolution can be minimal only if the multidegree of $b(p ; S \backslash\{q\})$ is a proper divisor the multidegree of $b(p ; S)$ for all $q$ in $S$.

By (a)

$$
\text { multideg } b(p ; S)=x_{A} y_{B} \quad \text { and } \quad \operatorname{multideg} b(p ; S \backslash\{q\})=x_{A} y_{C},
$$

where $A=\ell(p), B=\ell(p)^{c} \cup \bigcup_{r \in S} \ell(r)^{c}$ and $C=\ell(p)^{c} \cup \bigcup_{r \in S, r \neq q} \ell(r)^{c}$. Here, for any subset $F \subset P$, we set $F^{c}=P \backslash F$.

It follows that $v_{q}=1$ if and only if $\bigcap_{r \in S} \ell(r)=\bigcap_{r \in S, r \neq q} \ell(r)$. By Lemma1.1(iii) this is equivalent to saying that

$$
\bigwedge\{r: r \in S\}=\bigwedge\{r: r \in S, r \neq q\} .
$$

Hence if the resolution is minimal, we do not have equality in (3) for any $S \subset N(p)$ and any $q \in S$. In particular, for $S=N(p)$ we obtain the desired result.

(ii) $\Rightarrow$ (i): Let $b(p ; S)$ and $b(q ; T)$ be two basis elements with $|T|=|S|-1$. It suffices to show that in the following three cases the coefficient of $b(q ; T)$ in $\partial b(p ; S)$ is either 0 or a monomial $\neq 1$ :

- $p=q$ and $T \not \subset S$;

- $q<p$;

- $q \nless p$.

In the first case we show that multideg $b(p ; T)$ does not divide multideg $b(p ; S)$. Otherwise we would have that $\bigcup_{r \in T} \ell(r)^{c} \subseteq \bigcup_{r \in S} \ell(r)^{c}$. This would imply that $\bigcap_{r \in S} \ell(r) \subseteq \bigcap_{r \in T} \ell(r)$, which in turn would imply that

$$
\bigwedge\{r: r \in S\} \leq \bigwedge\{r: r \in T\}
$$

But then $\bigwedge\{r: r \in N(p)\}=\bigwedge\{r: r \in N(p) \backslash(T \backslash S)\}$, a contradiction.

In the second case we have multideg $b(p ; S)=x_{\ell(p)} y_{A}$ and multideg $b(q ; T)=$ $x_{\ell(q)} y_{B}$ for some $A$ and $B$. If multideg $b(q ; T)$ does not divide multideg $b(p ; S)$, then the coefficient of $b(q ; T)$ is 0 . Otherwise it is $x_{\ell(p) \backslash \ell(q)} y_{A \backslash B}$. Since $q<p$, this coefficient is not 1.

In the last case $\ell(q) \nsubseteq \ell \ell(p)$, and therefore multideg $b(q ; T)$ does not divide multideg $b(p ; S)$. Hence the coefficient of $b(q ; T)$ is 0 .

Corollary 2.2. If $\mathcal{L}$ is a meet-distributive meet-semilattice, then the finite multigraded free $S$-resolution given in Theorem 2.1 is minimal.

Proof. By definition meet-distributive meet-semilattices have the property that for any element $p \in \mathcal{L}$ the interval $[\bigwedge\{q: q \in N(p)\}, p]$ is a Boolean lattice (of rank $|N(p)|)$. This implies condition (b)(ii) in Theorem 2.1.

Note that condition (b)(ii) in Theorem 2.1 is satisfied for any meet-semilattice $\mathcal{L}$ for which $|N(p)| \leq 2$ for all $p \in \mathcal{L}$. Other examples can easily be constructed, as follows: let $\mathcal{L}$ be a meet-semilattice satisfying the condition (b)(ii), and let $p, q \in \mathcal{L}$ 
such that $q \in N(p)$. Let $\mathcal{L}^{\prime}$ be the meet-semilattice adding a new element $r$ with $q<r<p$. Then this new meet-semilattice again satisfies (b)(ii).

An example of such a meet-semilattice is

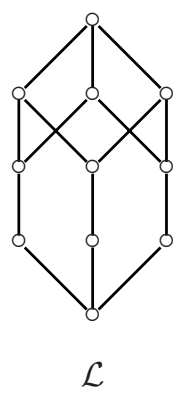

Observe that $\mathcal{L}$ is neither upper nor lower semimodular. The resolution of $H_{\mathcal{L}}$ is

$0 \longrightarrow S(-12) \longrightarrow S^{6}(-10) \longrightarrow S^{9}(-8) \oplus S^{6}(-7) \longrightarrow S^{11}(-6) \longrightarrow H_{\mathcal{L}} \longrightarrow 0$.

We close this section by discussing the regularity of $H_{\mathcal{L}}$. Recall that the regularity of a finitely generated graded $S$-module $M$ is defined to be

$$
\operatorname{reg} M=\max \left\{j: \beta_{i, i+j}(M) \neq 0 \text { for some } i\right\} .
$$

Corollary 2.3. Let $\mathcal{L}$ be a finite meet-semilattice and $P$ the poset of join irreducible elements in $\mathcal{L}$. Then:

(a) $\operatorname{reg}\left(H_{\mathcal{L}}\right) \leq|P|+\max _{\frac{p \in \mathcal{L}}{S \subset N(p)}}\{\operatorname{deg} p-\operatorname{deg} \bigwedge\{q: q \in S\}-|S|\}$;

(b) if $\mathcal{L}$ satisfies condition (b)(ii) in Theorem 2.1, then

$$
\operatorname{reg}\left(H_{\mathcal{L}}\right)=|P|+\max _{p \in \mathcal{L}}\{\operatorname{deg} p-\operatorname{deg} \bigwedge\{q: q \in N(p)\}-|N(p)|\} .
$$

Proof. Since $\mathbb{F}$ is a possibly non-minimal free resolution of $H_{\mathcal{L}}$, it follows that

$$
\operatorname{reg} H_{\mathcal{L}} \leq \max \{\operatorname{deg} b(p ; S)-|S|\},
$$

where the maximum is taken over all basis elements in the resolution.

By our computation in the proof of Theorem 2.1 one has

$$
\operatorname{deg} b(p ; S)-|S|=|P|+\operatorname{deg} p-\operatorname{deg} \bigwedge\{q: q \in S\}-|S| .
$$

This implies assertion (a).

If $\mathcal{L}$ satisfies condition (b)(ii) in Theorem 2.1, then our resolution is minimal and hence we have equality in formula (a). Moreover, if $S^{\prime} \subset S \subset N(p)$ with $|S|=\left|S^{\prime}\right|+1$, then

$$
\operatorname{deg} \bigwedge\{q: q \in S\}-\operatorname{deg} \bigwedge\left\{q: q \in S^{\prime}\right\} \geq 1 .
$$

Hence

$$
\bigwedge\{q: q \in S\}-\bigwedge\{q: q \in N(p)\} \geq|N(p)|-|S| .
$$




\section{The Resolution of $H_{\mathcal{L}}$ \\ FOR A MEET-DISTRIBUTIVE MEET-SEMILATTICE}

In this section we describe the differential $\partial$ in the graded minimal free resolution $\mathbb{F}$ of $H_{\mathcal{L}}$ when $\mathcal{L}$ is a meet-distributive meet-semilattice. One way to do this is to describe explicitly the comparison maps in the mapping cone construction in the proof of Theorem 2.1 .

Here we choose another way. Indeed, it turns out that if the meet-semilattice is meet-distributive, then the resolution given in Theorem 2.1 is a cellular resolution in the sense of Bayer and Sturmfels. The polyhedral cell complex $X$ supporting the resolution is the following: the cells are cubes whose vertices correspond to the elements of the Boolean intervals of $\mathcal{L}$. These intervals $[q, p]$ can be described as the sets $\mathcal{B}(p ; S)$, where $p \in \mathcal{L}, S \subset N(p)$ and $q=\bigwedge\{s: s \in S\}$. We claim that $X$ is in fact a polyhedral cell complex.

Let $\mathcal{C}$ be the cube corresponding to $[q, p]=\mathcal{B}(p ; S)$ and let $F$ be a face of $\mathcal{C}$. Then $F$ is a cube whose vertices correspond to the elements of an interval of $[q, p]$. Since every interval of the Boolean interval is again Boolean, it follows that $F$ is of the form $\mathcal{B}\left(p^{\prime} ; S^{\prime}\right)$.

In general, if $\left[q_{1}, p_{1}\right]$ and $\left[q_{2}, p_{2}\right]$ are intervals of a meet-semilattice, then the intersection $\left[q_{1}, p_{1}\right] \cap\left[q_{2}, p_{2}\right]$ is again an interval. Because, if $x$ and $y$ belong to $\left[q_{1}, p_{1}\right] \cap\left[q_{2}, p_{2}\right]$, then both $x \vee y$ and $x \wedge y$ belong to each of $\left[q_{1}, p_{1}\right]$ and $\left[q_{2}, p_{2}\right]$.

In particular, if $\mathcal{C}_{1}$ is a cube corresponding to $\left[q_{1}, p_{1}\right]=\mathcal{B}\left(p_{1} ; S_{1}\right)$ and $\mathcal{C}_{2}$ is a cube corresponding to $\left[q_{2}, p_{2}\right]=\mathcal{B}\left(p_{2} ; S_{2}\right)$, then $\mathcal{C}_{1} \cap \mathcal{C}_{2}$ is again a cube whose vertices correspond to the elements of an interval of $\left[q_{1}, p_{1}\right] \cap\left[q_{2}, p_{2}\right]$. Since $\left[q_{1}, p_{1}\right]$ is Boolean, its interval is again Boolean. Hence $\mathcal{C}_{1} \cap \mathcal{C}_{2}$ is again a cube whose vertices correspond to the elements of a Boolean interval.

The vertices of the cell complex $X$ are the elements of $\mathcal{L}$. Therefore the monomials $u_{p}$ with $p \in \mathcal{L}$ define a labelling on $X$. Now we consider the cellular resolution $\mathbb{F}_{X}$ supported on $X$. The basis elements of $F_{i}$ correspond to the $i$-faces of $X$. If $\mathcal{B}(p ; S)$ is such an $i$-face, we denote the corresponding basis element by $b(p ; S)$. Thus we see that we obtain in this particular case the same basis as in the resolution given in Theorem 2.1.

Let $P$ be the set of join-irreducible elements of $\mathcal{L}$. We extend the partial order on $P$ to a total order $<$. For a subset $T \subset P$ and $q \in P$ we set

$$
\sigma(q ; T)=|\{r \in T: r<q\}| .
$$

For each $q \in N(p)$, we have $|\ell(p) \backslash \ell(q)|=1$. We denote the unique element in $\ell(p) \backslash \ell(q)$ by $p \backslash q$. Furthermore, for any subset $S \subset N(p)$ we set $p \backslash S=\{p \backslash q: q \in S\}$, and we denote for any element $q \in \mathcal{L}$ and any subset $S \subset \mathcal{L}$ by $q \wedge S$ the set $\{q \wedge p: p \in S\}$.

With the notation introduced we now have

Theorem 3.1. The cellular complex $\mathbb{F}_{X}$ is a graded free $S$-resolution of $S / H_{\mathcal{L}}$, and for each $p \in \mathcal{L}$ and each $S \subset N(p)$, one has

$$
\partial(b(p ; S))=\sum_{q \in S}(-1)^{\sigma(p \backslash q ; p \backslash S)}\left(y_{p \backslash q} b(p ; S \backslash\{q\})-x_{p \backslash q} b(q ; q \wedge(S \backslash\{q\})) .\right.
$$

Before we give the proof of the theorem we first note that $q \wedge(S \backslash\{q\}) \subset N(q)$ for all $q \in S$. This is the case because by assumption $\mathcal{L}$ is meet-distributive, so that 
for any two distinct lower neighbors $q_{1}$ and $q_{2}$ of $p$, the element $q_{1} \wedge q_{2}$ is a lower neighbor of $q_{1}$ and $q_{2}$.

We also note that the differential defined in Theorem 3.1 is multi-homogeneous. To see this, recall that multideg $(b(p ; S))$ is the least common multiple of $u_{p}$ and all $u_{q}$ with $q \in S$. Since $u_{q}=y_{p \backslash q} u_{p} / x_{p \backslash q}$, we have $\operatorname{multideg}(b(p ; S \backslash\{q\}))=$ multideg $(b(p ; S)) / y_{p \backslash q}$, and $\operatorname{multideg}(b(q ; q \wedge(S \backslash\{q\})))=\operatorname{multideg}(b(p ; S)) / x_{p \backslash q}$. This shows that $\partial$ is indeed multi-homogeneous.

Proof of Theorem 3.1. In order to prove $\mathbb{F}_{X}$ is acyclic we use the criterion given in [1, Proposition 1.2]. We must show that $X_{\leq b}$ are acyclic for all $b$, where $X_{\leq b}$ denotes the cellular subcomplex consisting of those faces whose vertices have labels dividing $b$. It is easy to see that any of these subcomplexes is isomorphic to a meet-distributive meet-semilattice. Hence by [10, Proposition, p. 179] each $X_{\leq b}$ is acyclic.

Next we describe the differential. Considering the degrees of the basis elements in the resolution, the coefficients are determined up to signs. It remains to define the signs. For this purpose we have to define an incidence function $\varepsilon\left(\mathcal{B}\left(p ; S_{1}\right), \mathcal{B}\left(q ; S_{2}\right)\right)$ on pairs of faces taking values in $\{0,1-1\}$, such that $\varepsilon\left(\mathcal{B}\left(p ; S_{1}\right), \mathcal{B}\left(q ; S_{2}\right)\right)=0$, if $\mathcal{B}\left(q ; S_{2}\right)$ is not a face of $\mathcal{B}\left(p ; S_{1}\right)$, that $\varepsilon(p, \emptyset)=1$ and that

$$
\begin{aligned}
\varepsilon(\mathcal{B}(p ; S) & \left., \mathcal{B}\left(q_{1} ; S_{1}\right)\right) \varepsilon\left(\mathcal{B}\left(q_{1} ; S_{1}\right), \mathcal{B}\left(r ; S^{\prime}\right)\right) \\
& +\varepsilon\left(\mathcal{B}(p ; S), \mathcal{B}\left(q_{2} ; S_{2}\right)\right) \varepsilon\left(\mathcal{B}\left(q_{2} ; S_{2}\right), \mathcal{B}\left(r ; S^{\prime}\right)\right)=0
\end{aligned}
$$

where $\mathcal{B}\left(r ; S^{\prime}\right)$ is a face of codimension 2 in $\mathcal{B}(p ; S)$ and where $\mathcal{B}\left(q_{1} ; S_{1}\right)$ and $\mathcal{B}\left(q_{2} ; S_{2}\right)$ are faces of codimension 1 in $\mathcal{B}(p ; S)$ containing $\mathcal{B}\left(r ; S^{\prime}\right)$.

The faces of codimension 1 of $\mathcal{B}(p ; S)$ are of the form

$$
\mathcal{B}(p ; S \backslash\{q\}) \text { and } \mathcal{B}(q ; q \wedge(S \backslash\{q\})) \text {. }
$$

We set

$$
\varepsilon(\mathcal{B}(p ; S), \mathcal{B}(p ; S \backslash\{q\}))=(-1)^{\sigma(p \backslash q ; p \backslash S)}
$$

and

$$
\varepsilon(\mathcal{B}(p ; S), \mathcal{B}(q ; q \wedge S \backslash\{q\}))=-(-1)^{\sigma(p \backslash q ; p \backslash S)} .
$$

A routine but tedious calculation guaranties that $\varepsilon$ is indeed an incidence function.

It would be desirable to also have an explicit description of the differentials for the resolution of $H_{\mathcal{L}}$ when $\mathcal{L}$ is a meet-irredundant meet-semilattice. Quite generally, according to the iterated mapping cone construction described in Theorem 2.1, the differentials in the resolution of $H_{\mathcal{L}}$ for a meet-irredundant meet-semilattice is of the form

$$
\partial(b(p ; S))=\sum_{q \in S}(-1)^{\sigma(p \backslash q ; p \backslash S)} y_{p \backslash q} b(p ; S \backslash\{q\})+\sum_{t \in[r, p], t \neq p} c_{t} b\left(t ; S_{t}\right),
$$

where

(1) $r$ is the meet of all elements in $S$,

(2) $c_{t}=\lambda_{t} v_{t}$ with $\lambda_{t} \in K$ and $v_{t}$ is the monomial whose multidegree is multideg $(b(p ; S))$ - multideg $\left(b\left(t ; S_{t}\right)\right)$,

(3) $S_{t}$ is a set of lower neighbors of $t$ in the interval $[r, p]$ with $\left|S_{t}\right|=|S|-1$. 
For example consider the following meet irredundant meet-semilattice:

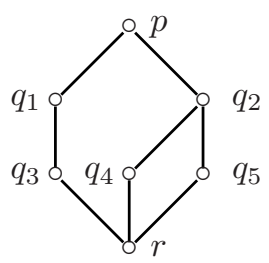

$\mathcal{L}$

whose poset of join irreducible elements is

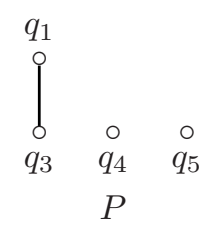

It is easy to see that in this case there are two, equally natural choices, to define $\partial\left(b\left(p ;\left\{q_{1}, q_{2}\right\}\right)\right.$, namely:

$$
\begin{aligned}
\partial\left(b\left(p ;\left\{q_{1}, q_{2}\right\}\right)=-\right. & y_{1} y_{3} b\left(p ;\left\{q_{1}\right\}\right)+y_{4} y_{5} b\left(p ;\left\{q_{2}\right\}\right)-x_{4} x_{5} y_{3} b\left(q_{1} ;\left\{q_{3}\right\}\right) \\
& -x_{1} x_{4} x_{5} b\left(q_{3} ;\{r\}\right)+x_{1} x_{3} y_{4} b\left(q_{2} ;\left\{q_{4}\right\}\right)+x_{1} x_{3} x_{5} b\left(q_{4} ;\{r\}\right),
\end{aligned}
$$

or,

$$
\begin{aligned}
\partial\left(b\left(p ;\left\{q_{1}, q_{2}\right\}\right)=-\right. & y_{1} y_{3} b\left(p ;\left\{q_{1}\right\}\right)+y_{4} y_{5} b\left(p ;\left\{q_{2}\right\}\right)-x_{4} x_{5} y_{3} b\left(q_{1} ;\left\{q_{3}\right\}\right) \\
& -x_{1} x_{4} x_{5} b\left(q_{3} ;\{r\}\right)+x_{1} x_{3} y_{5} b\left(q_{2} ;\left\{q_{5}\right\}\right)+x_{1} x_{3} x_{4} b\left(q_{5} ;\{r\}\right) .
\end{aligned}
$$

Here we wrote for simplicity $x_{i}$ and $y_{i}$ instead of $x_{q_{i}}$ and $y_{q_{i}}$, respectively.

\section{On the Alexander dual of $H_{\mathcal{L}}$}

For convenience we introduce the following notation: let $I$ be a squarefree monomial ideal. Then $I=I_{\Delta}$ for some simplicial complex $\Delta$, and we write $I^{*}$ for $I_{\Delta^{\vee}}$. Here, as before, $\Delta^{\vee}$ is the Alexander dual of the simplicial complex $\Delta$.

Let $\mathcal{L}$ be a distributive lattice. In particular $\mathcal{L}$ is a poset, and we may consider a poset ideal $\mathcal{I} \subset \mathcal{L}$. Note that any poset ideal $\mathcal{I}$ of $\mathcal{L}$ is a (special) meet-semilattice.

Let $p \in \mathcal{L}$; then the poset ideal

$$
\mathcal{I}_{p}=\{q \in \mathcal{L}: q \nsupseteq p\}
$$

is called 1-cogenerated. It is clear that for any poset ideal $\mathcal{I}$ we have

$$
\mathcal{I}=\bigcap_{p \in \mathcal{L} \backslash \mathcal{I}} \mathcal{I}_{p}
$$

We set $H_{\mathcal{I}}=\left(\left\{u_{q}: q \in \mathcal{I}\right\}\right)$. Then

Lemma 4.1. For any poset ideal $\mathcal{I} \in \mathcal{L}$ we have

$$
H_{\mathcal{I}}=\bigcap_{q \in \mathcal{L} \backslash \mathcal{I}} H_{\mathcal{I}_{q}} \quad \text { and } \quad H_{\mathcal{I}}^{*}=\sum_{q \in \mathcal{L} \backslash \mathcal{I}} H_{\mathcal{I}_{q}}^{*}
$$


Proof. In order to prove the first equation, it suffices to show that if $\mathcal{J}$ and $\mathcal{K}$ are two poset ideals in $\mathcal{L}$, and $\mathcal{I}=\mathcal{J} \cap \mathcal{K}$, then $H_{\mathcal{I}}=H_{\mathcal{J}} \cap H_{\mathcal{K}}$. It is clear that $H_{\mathcal{I}} \subset H_{\mathcal{J}} \cap H_{\mathcal{K}}$. Let $m \in H_{\mathcal{J}} \cap H_{\mathcal{K}}$ be a monomial. Then there exist $p \in \mathcal{J}$ and $q \in \mathcal{K}$ such that $u_{p} \mid m$ and $u_{q} \mid m$. Let $t=p \wedge q$. Since $\mathcal{L}$ is distributive, we have $u_{t}=x_{\ell(p) \cap \ell(q)} y_{P \backslash(\ell(p) \cap \ell(q))}=x_{\ell(p) \cap \ell(q)} y_{(P \backslash \ell(p)) \cup(P \backslash \ell(q))}$; hence $u_{t} \mid m$. Since $t \leq p$ and $t \leq q$, it follows that $t \in \mathcal{J} \cap \mathcal{K}=\mathcal{I}$. Therefore, $m \in H_{\mathcal{I}}$.

Let $P$ be a monomial prime ideal. Then $\bigcap_{q \in \mathcal{L} \backslash \mathcal{I}} H_{\mathcal{I}_{q}} \subset P$ if and only if $H_{\mathcal{I}_{q}} \subset P$ for some $q$. Hence the second assertion follows from (1).

Theorem 4.2. Let $\mathcal{L}$ be a finite distributive lattice, $P \subset \mathcal{L}$ the poset of join irreducible elements of $\mathcal{L}$, and $\mathcal{I} \subset \mathcal{L}$ a poset ideal of $\mathcal{L}$. Then

$$
H_{\mathcal{I}}^{*}=\left(H_{\mathcal{L}}^{*},\left\{\prod_{r \in G(\ell(q))} y_{r}: q \in \mathcal{L} \backslash \mathcal{I}\right\}\right),
$$

where $G(\ell(q))$ is the set of generators of the poset ideal $\ell(q) \subset P$.

Proof. By using Lemma 4.1 it suffices to prove the theorem for a 1-cogenerated poset ideal $\mathcal{I}_{p}$. In this case what we must prove is

$$
H_{\mathcal{I}_{p}}^{*}=\left(H_{\mathcal{L}}^{*},\left\{\prod_{r \in G(\ell(q))} y_{r}: q \geq p\right\}\right) .
$$

Let $x_{A} y_{B}$ be a squarefree monomial with $A, B \subset P$. Then $x_{A} y_{B} \in H_{\mathcal{I}_{p}}^{*}$ if and only if $A \cap \ell(r) \neq \emptyset$, or $B \cap \ell(r)^{c} \neq \emptyset$ for all $r \geq p$.

Let $T=\left(H_{\mathcal{L}}^{*},\left\{\prod_{r \in G(\ell(q))} y_{r}: q \geq p\right\}\right)$. We first show that $T \subset H_{\mathcal{I}_{p}}^{*}$. Since $H_{\mathcal{I}_{p}} \subset H_{\mathcal{L}}$, it follows that $H_{\mathcal{L}}^{*} \subset H_{\mathcal{I}_{p}}^{*}$. Moreover, suppose that for some $q \geq p$ the monomial $\prod_{r \in G(\ell(q))} y_{r}$ does not belong to $H_{\mathcal{I}_{p}}^{*}$. Then there exists $t \geq p$ such that $G(\ell(q)) \cap \ell(t)^{c}=\emptyset$, equivalently $G(\ell(q)) \subset \ell(t)$. Hence $\ell(q) \subset \ell(t)$. However, since $q \geq p$, we have $\ell(p) \subset \ell(q)$, so that $\ell(p) \subset \ell(q)$, a contradiction.

It remains to show that $H_{\mathcal{I}_{p}}^{*} \subset T$.

Suppose $B=\emptyset$. Then $A \cap \ell(\hat{0})=\emptyset$ since $\ell(\hat{0})=\emptyset$ and also $B \cap \ell(\hat{0})^{c}=\emptyset$, a contradiction.

Suppose $A=\emptyset$. Let $\Delta^{\vee}$ denote the simplicial complex whose Stanley-Reisner ideal is equal to $H_{\mathcal{I}_{p}}^{*}$ and let $\Delta_{y}^{\vee}$ denote the restriction of $\Delta^{\vee}$ on the vertex set $\left\{y_{t}: t \in P\right\}$. Then the facets of $\Delta_{y}^{\vee}$ are $\left\{y_{t}: t \in \mathcal{I}\right\}$, where $\mathcal{I}$ is a maximal poset ideal of $P$ which does not contain $\ell(p)$. Such a poset ideal is of the form $P \backslash\{t \in P: t \geq h\}$ with $h \in G(\ell(p))$. If $y_{B}$ belongs to $H_{\mathcal{I}_{p}}^{*}$, then $B$ is contained in no facet of $\Delta_{y}^{\vee}$. Hence, for each $h \in G(\ell(p))$, there is $h^{\prime} \in P$ with $h^{\prime} \geq h$ such that $h^{\prime} \in B$. Let $\mathcal{I}_{0}$ denote the poset ideal of $P$ consisting of all $t \in P$ with $t \leq h^{\prime}$ for some $h \in G(\ell(p))$. Let $q \in \mathcal{L}$ with $\ell(q)=\mathcal{I}_{0}$. It then follows that $\prod_{r \in G(\ell(q))} y_{r}$ divides $y_{B}$.

Finally we consider the case that $A \neq \emptyset$ and $y_{B} \notin H_{\mathcal{I}_{p}}^{*}$. We will show that in this case $x_{A} y_{B} \in H_{\mathcal{L}}^{*}$. In fact, since $y_{B} \notin H_{\mathcal{I}_{p}}^{*}$, there exists $r \nsucceq p$ such that $B \cap \ell(r)^{c}=\emptyset$, equivalently $B \subset \ell(r)$. Let $(B) \subset P$ be the poset ideal generated by $B$. Then there exists $t \in \mathcal{L}$ such that $\ell(t)=(B)$. Since $\ell(t)=(B) \subset \ell(r)$ it follows that $t \leq r$, and hence $t \in \mathcal{I}_{p}$.

Suppose $x_{A} y_{B} \notin H_{\mathcal{L}}^{*}$; then $a \not \leq b$ for all $a \in A$ and $b \in B$. This implies that $A \cap(B)=A \cap \ell(t)=\emptyset$. This is a contradiction because also $B \cap \ell(t)^{c}=\emptyset$. 
Let $\Delta$ be the simplicial complex with $I(\Delta)=H_{\mathcal{I}}^{*}$. Then Theorem 4.2 implies that $\Delta$ satisfies the following conditions: there is a decomposition $V \cup V^{\prime}$ of the vertex set of $\Delta$ with $V \cap V^{\prime}=\emptyset$ and $|V|=\left|V^{\prime}\right|$, such that

(1) there is no $F \in \mathcal{F}(\Delta)$ with $F \subset V$,

(2) $G=\left\{F \in \mathcal{F}(\Delta): F \cap V \neq \emptyset, \quad F \cap V^{\prime} \neq \emptyset\right\}$ is a Cohen-Macaulay bipartite graph with no isolated vertex.

In fact, the facets in (1) correspond to the monomials $\left\{\prod_{r \in G(\ell(q))} y_{r}: q \in \mathcal{L} \backslash \mathcal{I}\right\}$, while the bipartite graph $G$ in (2) corresponds to $H_{\mathcal{L}}^{*}$; see [6, Theorem 3.4].

The question is, which simplicial complexes satisfying (1) and (2) come from a poset ideal of a distributive lattice? In order to describe the result we need some more definitions and notation: let $G$ be a finite graph on the vertex set $[n]$. The edge ideal of $G$ is the ideal $I(G)$ of $S=K\left[x_{1}, \ldots, x_{n}\right]$ generated by those monomials $x_{i} x_{j}$ such that $\{i, j\}$ is an edge of $G$. The graph $G$ is called Cohen-Macaulay over $K$ if $S / I(G)$ is Cohen-Macaulay.

Recall from [6. Theorem 3.4] that if $G$ is a Cohen-Macaulay bipartite graph on the vertex set $V \cup V^{\prime}$ with $V \cap V^{\prime}=\emptyset$ and $|V|=\left|V^{\prime}\right|$, then there exists a partial order $<$ on $V$ such that the distributive lattice $\mathcal{J}(P)$ with $P=(V,<)$ satisfies $H_{\mathcal{J}(P)}^{*}=I(G)$. We write $\mathcal{L}(G)$ for the distributive lattice $\mathcal{J}(P)$.

Theorem 4.3. Let $\Delta$ be a simplicial complex satisfying the above conditions (1) and (2). Then the following conditions are equivalent:

(a) $S / I(\Delta)$ is Cohen-Macaulay;

(b) The simplicial complex $\Gamma$ with $I_{\Gamma}=I(\Delta)$ is pure;

(c) There exists a poset ideal $\mathcal{I} \subset \mathcal{L}(G)$ containing all join-irreducible elements of $\mathcal{L}(G)$ such that $H_{\mathcal{I}}^{*}=I(\Delta)$.

The following pictures show examples of simplicial complexes satisfying the conditions (1) and (2):

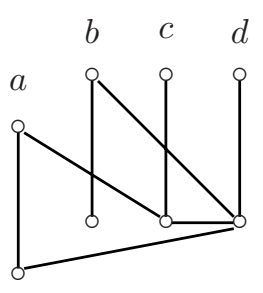

$\Delta_{1}$

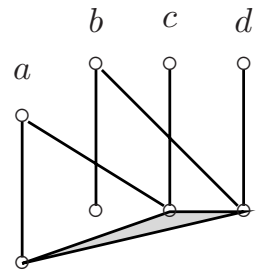

$\Delta_{2}$

The facet ideal of the simplicial complex $\Delta_{1}$ is Cohen-Macaulay, and that of $\Delta_{2}$ is not Cohen-Macaulay. In fact, the distributive lattice $\mathcal{L}$ and its poset $P$ of join irreducible elements corresponding to the bipartite graph in $\Delta$ and $\Delta^{\prime}$ is in both cases as shown in the following picture.

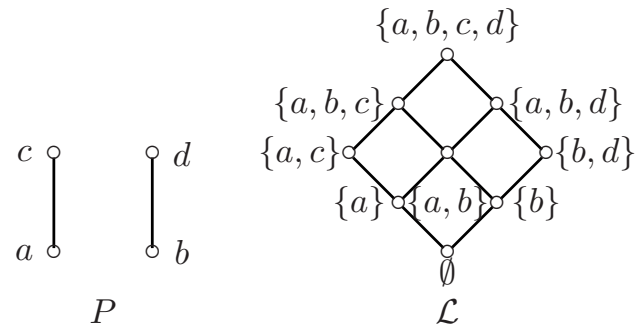


The simplicial complex $\Delta$ corresponds to the ideal

$$
\mathcal{I}=\{\emptyset,\{a\},\{b\},\{a, b\},\{a, c\},\{b, d\},\{a, b, c\}\} .
$$

Since all poset ideals of $\mathcal{L}$ are generated by at most two elements, it follows from Theorem 4.2 that the simplicial complex $\Delta^{\prime}$ cannot correspond to any poset ideal in $\mathcal{L}$. Therefore, by Theorem 4.3 it cannot be Cohen-Macaulay.

Proof of Theorem 4.3. Since every Cohen-Macaulay simplicial complex is pure, one has $(\mathrm{a}) \Rightarrow(\mathrm{b})$. Moreover, since Theorem 1.3 guarantees that $H_{\mathcal{I}}$ has a linear resolution, it follows from Lemma 1.5 that $(\mathrm{c}) \Rightarrow$ (a).

We now prove that $(\mathrm{b}) \Rightarrow(\mathrm{c})$. Let $V=\left\{x_{1}, \ldots, x_{n}\right\}$ and $V^{\prime}=\left\{y_{1}, \ldots, y_{n}\right\}$. Since $\Gamma$ is pure and since $V$ is a facet of $\Gamma$, it follows that each facet of $\Gamma$ is a facet of $\Gamma_{0}$, where $\Gamma_{0}$ is a simplicial complex on $V \cup V^{\prime}$ with $I_{\Gamma_{0}}=I(G)$. In other words, each minimal non-face of $\Gamma^{\vee}$ is a minimal non-face of $\Gamma_{0}^{\vee}$. Thus we may regard the minimal set $\mathcal{I}^{b}$ of monomial generators of $I_{\Gamma} \vee$ as a subset of $\mathcal{L}(G)$. Now, what we must prove is that $\mathcal{I}^{b}$ is a poset ideal of $\mathcal{L}(G)=\mathcal{J}(P)$, where $P=(V,<)$ is the poset consisting of all join-irreducible elements of $\mathcal{L}(G)$. Suppose, on the contrary, that $\mathcal{I}^{b}$ is not a poset ideal, and choose two elements $\delta$ and $\xi$ of $\mathcal{L}(G)$ with $\delta \in \mathcal{I}^{\text {b }}$ and $\xi \notin \mathcal{I}^{\text {b }}$ such that $\delta$ covers $\xi$ in $\mathcal{L}(G)$. To simplify the notation, we will assume that $\delta=\left\{x_{1}, \ldots, x_{k}\right\}$ and $\xi=\left\{x_{1}, \ldots, x_{k-1}\right\}$. Thus $\left\{y_{1}, \ldots, y_{k}, x_{k+1}, \ldots, x_{n}\right\}$ is a facet of $\Gamma$ and $\left\{y_{1}, \ldots, y_{k-1}, x_{k}, x_{k+1}, \ldots, x_{n}\right\}$ is not a facet of $\Gamma$. Thus there is a monomial generator $u$ of $I(\Delta)$ which divides $y_{1} \cdots y_{k-1} x_{k} x_{k+1} \cdots x_{n}$. However, since $\left\{y_{1}, \ldots, y_{k-1}, x_{k+1}, \ldots, x_{n}\right\}$ is a face of $\Gamma$, it follows that the variable $x_{k}$ must appear in the support of $u$. Hence $u=x_{k} y_{j}$ with $1 \leq j \leq k-1$. Then [ 6 , Theorem 3.4] says that $x_{k}<x_{j}$ in $P$. This is impossible, since $\xi$ is a poset ideal of $\mathcal{L}(G)$. Consequently, it turns out that $\mathcal{I}^{\text {b }}$ is a poset ideal of $\mathcal{L}(G)$.

Finally, in the case that $\mathcal{I}^{\text {b }}$ does not contain of a join-irreducible element $x_{i}$ of $\mathcal{L}(G)$, the vertex $y_{i}$ belongs to all facets of $\Gamma$. This is impossible, since $G$ possesses no isolated vertex. This completes the proof of $(b) \Rightarrow(c)$.

Corollary 4.4. Let $\Delta$ be a simplicial complex on the vertex set $V=\left\{v_{1}, \ldots, v_{n}\right\}$, and let $W=\left\{w_{1}, \ldots, w_{n}\right\}$ be a vertex set with $W \cap V=\emptyset$. Let $\Gamma$ be the simplicial complex on the vertex set $V \cup W$ whose facets are those of $\Delta$ and all the edges $\left\{v_{i}, w_{i}\right\}$ for $i=1, \ldots, n$. Then the facet ideal of $\Gamma$ is Cohen-Macaulay.

Proof. Our work is to show that the simplicial complex $\Sigma$ with $I_{\Sigma}=I(\Gamma)$ is pure. Let $F=\left\{v_{i}: i \in A\right\} \cup\left\{w_{j}: j \in B\right\}$ be a face of $\Sigma$; then $A \cap B=\emptyset$. If $A \cup B \neq[n]$, then $F \cup\left\{w_{i}: i \in[n] \backslash(A \cup B)\right\}$ is a face of $\Sigma$. Thus all facets of $\Sigma$ have the cardinality $n$. Hence $\Sigma$ is pure, as desired.

Corollary 4.4 can be rephrased topologically: Fix a facet $V$ in the boundary $K$ of a cross-polytope. Then for any collection $\Delta$ of subfaces of $V$, if one deletes these faces (and all faces containing them) from $K$, the result is Cohen-Macaulay.

The results of Theorem 1.3 and Theorem 4.2 can be extended as follows. Let $P$ be a poset. Recall that a poset coideal of $P$ is a subset $J \subset P$ with the property that for each $p \in J$ and each $q \in P$ with $q \geq p$ one has $q \in J$. The minimal elements in $J$ are called the cogenerators. The set of cogenerators of $J$ will be denoted by $G(J)$.

Now let $\mathcal{L}$ be a finite distributive lattice, let $\mathcal{I} \subset \mathcal{L}$ be a poset ideal, and let $\mathcal{J}$ be a poset coideal in $\mathcal{L}$. We write $H_{(\mathcal{I}, \mathcal{L})}\left(\right.$ resp. $\left.H_{(\mathcal{J}, \mathcal{L})}\right)$ for the ideal of $S$ generated by 
those monomials $u_{q}$ with $q \in \mathcal{I}$ (resp. $q \in \mathcal{J}$ ). Then both $H_{(\mathcal{I}, \mathcal{L})}$ and $H_{(\mathcal{J}, \mathcal{L})}$ have linear resolutions. Both ideals are isomorphic to the ideal of a meet-distributive meet-semilattice, namely $\mathcal{I}$ and the dual of $\mathcal{J}$. Therefore, Theorem 1.3 implies that these ideals have a linear resolution.

What can be said about $H_{(\mathcal{I}, \mathcal{L})} \cap H_{(\mathcal{J}, \mathcal{L})}$ ? The reader might expect that this ideal again has a linear resolution. However this is not the case. For example, consider the Boolean lattice $\mathcal{B}_{3}$ of $\operatorname{rank} 3$, and let $\mathcal{I}=\mathcal{B}_{3} \backslash\{\hat{1}\}$ and $\mathcal{J}=\mathcal{B}_{3} \backslash\{\hat{0}\}$. Then $H_{(\mathcal{I}, \mathcal{L})} \cap H_{(\mathcal{J}, \mathcal{L})}$ does not have a linear resolution.

However in the positive direction we have

Proposition 4.5. Let $\mathcal{I}$ be a poset ideal and $\mathcal{J}$ a poset coideal in $\mathcal{L}$. Then

(a) $\operatorname{rank} \mathcal{L} \leq \operatorname{reg}\left(H_{(\mathcal{I}, \mathcal{L})} \cap H_{(\mathcal{J}, \mathcal{L})}\right) \leq \operatorname{rank} \mathcal{L}+1$, if $\mathcal{L}=\mathcal{I} \cup \mathcal{J}$.

(b) $\left(H_{(\mathcal{I}, \mathcal{L})} \cap H_{(\mathcal{J}, \mathcal{L})}\right)^{*}=\left(H_{\mathcal{L}}^{*},\left\{\prod_{r \in G(\ell(q))} y_{r}: q \in \mathcal{L} \backslash \mathcal{I}\right\},\left\{\prod_{r \in G\left(\ell(q)^{c}\right)} x_{r}: q \in\right.\right.$ $\mathcal{L} \backslash \mathcal{J}\})$.

Proof. (a) Consider the long exact Tor-sequence

$$
\begin{array}{r}
\cdots \rightarrow \operatorname{Tor}_{i+1}\left(K, H_{(\mathcal{I}, \mathcal{L})}+H_{(\mathcal{J}, \mathcal{L})}\right) \rightarrow \operatorname{Tor}_{i}\left(K, H_{(\mathcal{I}, \mathcal{L})} \cap H_{(\mathcal{J}, \mathcal{L})}\right) \\
\rightarrow \operatorname{Tor}_{i}\left(K, H_{(\mathcal{I}, \mathcal{L})}\right) \oplus \operatorname{Tor}_{i}\left(K, H_{(\mathcal{J}, \mathcal{L})}\right) \cdots
\end{array}
$$

arising from the short exact sequence

$$
0 \Rightarrow H_{(\mathcal{I}, \mathcal{L})} \cap H_{(\mathcal{J}, \mathcal{L})} \longrightarrow H_{(\mathcal{I}, \mathcal{L})} \oplus H_{(\mathcal{J}, \mathcal{L})} \longrightarrow H_{(\mathcal{I}, \mathcal{L})}+H_{(\mathcal{J}, \mathcal{L})} \longrightarrow 0 .
$$

Since $H_{\mathcal{L}}=H_{(\mathcal{I}, \mathcal{L})}+H_{(\mathcal{J}, \mathcal{L})}$, the ideals $H_{(\mathcal{I}, \mathcal{L})}, H_{(\mathcal{J}, \mathcal{L})}$ and $H_{(\mathcal{I}, \mathcal{L})}+H_{(\mathcal{J}, \mathcal{L})}$ have a linear resolution by Theorem 1.3 . It follows that

$$
\operatorname{Tor}_{i}\left(K, H_{(\mathcal{I}, \mathcal{L})}\right)_{j}=\operatorname{Tor}_{i}\left(K, H_{(\mathcal{J}, \mathcal{L})}\right)_{j}=0
$$

for $j \neq i+\operatorname{rank} \mathcal{L}$, and $\operatorname{Tor}_{i+1}\left(K, H_{(\mathcal{I}, \mathcal{L})}+H_{(\mathcal{J}, \mathcal{L})}\right)_{j}=0$ for $j \neq i+1+\operatorname{rank} \mathcal{L}$. Thus the assertion follows from the long exact Tor-sequence. 4.2.

(b) Since $\left(H_{(\mathcal{I}, \mathcal{L})} \cap H_{(\mathcal{J}, \mathcal{L})}\right)^{*}=H_{(\mathcal{I}, \mathcal{L})}^{*}+H_{(\mathcal{J}, \mathcal{L})}^{*}$, the claim follows Theorem

Consider the following example.

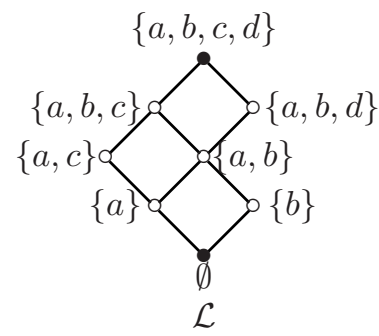

Here we take in $\mathcal{L}$ the poset ideal

$$
\mathcal{I}=\{\emptyset,\{a\},\{b\},\{a, b\},\{a, c\},\{a, b, c\},\{a, b, d\}\}
$$

and the poset coideal

$$
\mathcal{J}=\{\{a\},\{b\},\{a, b\},\{a, c\},\{a, b, c\},\{a, b, d\},\{a, b, c, d\}\} .
$$

Then $H_{(\mathcal{I}, \mathcal{L})} \cap H_{(\mathcal{J}, \mathcal{L})}=(a v w x, b u w x, a c v x, a b w x, a b c x, a b d w)$. Thus this intersection is generated by all generators of $H_{\mathcal{L}}$ except $u_{\hat{0}}$ and $u_{\hat{1}}$, as indicated in the picture. The resolution of $H_{(\mathcal{I}, \mathcal{L})} \cap H_{(\mathcal{J}, \mathcal{L})}$ is linear, namely

$$
0 \longrightarrow S(-6) \longrightarrow S(-5)^{6} \longrightarrow S(-4)^{6} \longrightarrow H_{(\mathcal{I}, \mathcal{L})} \cap H_{(\mathcal{J}, \mathcal{L})} \longrightarrow 0 .
$$


Quite generally it would be interesting to know when $H_{(\mathcal{I}, \mathcal{L})} \cap H_{(\mathcal{J}, \mathcal{L})}=H_{(\mathcal{I} \cap \mathcal{J}, \mathcal{L})}$, and when an ideal of the form $H_{(\mathcal{I} \cap \mathcal{J}, \mathcal{L})}$ has a linear resolution. Of particular interest are the following cases:

(1) $H=\left(\left\{u_{p}\right\}_{p \in \mathcal{L} \backslash\{\hat{0}, \hat{1}\}}\right)$;

(2) $H=\left(\left\{u_{p}: r \leq \operatorname{rank} p \leq s\right\}\right)$ for some $r$ and $s$ with $0<r \leq s<\operatorname{rank} \mathcal{L}$.

\section{REFERENCES}

[1] D. Bayer and B. Sturmfels, Cellular resolutions of monomial modules, J. Reine Angew. Math. 502 (1998), 123-140. MR:1647559 (99g:13018)

[2] W. Bruns and J. Herzog, "Cohen-Macaulay rings," Revised Edition, Cambridge University Press, 1996. MR1251956 (95h:13020)

[3] J. Eagon and V. Reiner, Resolutions of Stanley-Reisner rings and Alexander duality, J. Pure Appl. Algebra 130 (1998), 265-275. MR1633767(99h:13017)

[4] P. Edelman, Abstract convexity and meet-distributive lattices, in "Combinatorics and ordered sets" (Arcata, Calif., 1985), Contemp. Math. 57, 127-150, Amer. Math. Soc., Providence, RI, 1986. MR0856235 (87m:52003)

[5] D. Eisenbud, "Commutative Algebra with a view to Algebraic geometry", Springer-Verlag, 1995. MR1322960(97a:13001)

[6] J. Herzog and T. Hibi, Distributive Lattices, Bipartite Graphs and Alexander Duality, J. Alg. Combin. 22 (2005), 289-303. MR 2181367

[7] J. Herzog, T. Hibi and X. Zheng, Dirac's theorem on chordal graphs and Alexander duality, European J. Combin. 25 (2004), 949-960. MR2083448

[8] T. Hibi, Distributive lattices, affine semigroup rings and algebras with straightening laws, in "Commutative Algebra and Combinatorics" (M. Nagata and H. Matsumura, Eds.), Advanced Studies in Pure Math., Volume 11, North-Holland, Amsterdam, 1987, pp. 93-109. MR0951198 (90b:13024)

[9] T. Hibi, "Algebraic Combinatorics on Convex Polytopes," Carslaw, Glebe, N.S.W., Australia, 1992.

[10] R. P. Stanley, "Enumerative Combinatorics, Volume I," Wadsworth and Brooks/Cole, Monterey, CA, 1986. MR0847717 (87j:05003)

[11] R. P. Stanley, "Combinatorics and Commutative Algebra," Second Edition, Birkhäuser, Boston, MA, 1996. MR 1453579(98h:05001)

Fachbereich Mathematik und Informatik, Universität Duisburg-Essen, 45117 Essen, GERMANY

E-mail address: juergen.herzog@uni-essen.de

Department of Pure and Applied Mathematics, Graduate School of Information Science and Technology, Osaka University, Toyonaka, Osaka 560-0043, Japan

E-mail address: hibi@math.sci.osaka-u.ac.jp

Fachbereich Mathematik und Informatik, Universität Duisburg-Essen, 45117 Essen, Germany

E-mail address: xinxian.zheng@uni-essen.de 